\title{
Spectrum of radiological presentations among the patients of intracranial metastasis
}

\author{
Shilpi Muchhoria', Sourabh Dixit², Sandeep BV³ , Suniti Kumar Saha ${ }^{4}$, \\ Abhishek Nadkarni ${ }^{5}$, Debjyoti Pathak ${ }^{5}$ \\ ${ }^{1}$ Consultant Diagnostic and Interventional Radiologist, Bhopal Memorial Hospital and Research Centre (BMHRC) \\ Bhopal, Madhya Pradesh, India, ${ }^{2}$ Consultant Neurosurgeon, Bhopal Memorial Hospital and Research Centre (BMHRC) \\ Bhopal, Madhya Pradesh, India, ${ }^{3}$ Assistant Professor Neurosurgery, Vydehi Medical College, Karnataka Bengaluru, \\ India, ${ }^{4}$ Professor and Head of Department, Department of Neurosurgery, Nil Ratan Sircar Medical college and Hospital, \\ Kolkata, West Bengal, India, ${ }^{5}$ Post Doctoral Trainee, Department Of Neurosurgery, Nil Ratan Sircar Medical College \\ and Hospital,Kolkata, West Bengal, India
}

Background: Brain metastasis has been the most common primary intracranial space occupying lesion (ICSOL) in adult patients. The main systemic malignancies presenting with high tendency to invade brain parenchyma are -lung, breast, melanoma, renal and colon cancers etc. Aims and Objectives: 1. To study the various radiological presentations in cases of intracranial metatstasis. 2. To assess the radiological presentations to differentiate metastasis from other differential diagnosis. Materials and Methods: The present cross sectional study was carried out amongst 32 patients who had been visited tertiary care hospital in Kolkata with radiological features and histopathology suggestive of metastatic lesion. Results: The mean age of the subjects was 46.56 years and the maximum number of cases presented in $5^{\text {th }}$ and $6^{\text {th }}$ decades of life. CT Scan showed $75 \%$ patient had metastatic lesion appearing hyperdense on plain study and $25 \%$ had lesion hypodense on plain CT Scan. On T1 weighted images- In $75 \%$ of patient the metastatic lesions were hypointense to brain parenchyma and in $12.5 \%$ they were isointense and $12.5 \%$ they were hyperintense. T2 weighted images- In $62.5 \%$ of patient the metastatic lesions were hyperintense to brain parenchyma and in $25 \%$ patients they were isointense and in $12.5 \%$ they were hypointense. Contrast Images $-37.5 \%$ of patients had homogenous enhancement and $50 \%$ had heterogeneous enhancement and $12.5 \%$ had ring shaped enhancement. In Biopsy about $75 \%$ the metastatic lesion turned out to be metastatic carcinoma. Conclusion: A thorough and systematic approach to radiological investigation is crucial for proper identification of intracranial metastatic lesions.

Key words: Melanoma; Intracranial metastasis; Intracranial space occupying lesion
Access this article online

Website:

http://nepjol.info/index.php/AJMS

DOI: /10.3126/ajms.v10i4.23781

E-ISSN: 2091-0576

P-ISSN: $2467-9100$

\section{INTRODUCTION}

Brain metastasis has been found in 9-17\% cases of systemic malignancies either as intial manifestation or along with other visceral involvemnts. ${ }^{1,2}$ Brain metastasis has been the most common primary intracranial space occupying (ICSOL) lesion in adult patients. The main systemic malignancies presenting with high tendency to invade brain parenchyma are -lung, breast, melanoma, renal and colon cancers etc. ${ }^{2}$
Brain imaging advancements have led to increased number of detected cases of ICSOL and also Metastasis. ${ }^{3}$ Magnetic resonance imaging (MRI) is the back balance of the investigations. MRI is not only important for diagnosis but also for proper planning of surgical treatment. A number of studies have concluded that radiological features can assist in assessment of prognosis in patients of brain metastasis ${ }^{4}$. The current study was undertaken among the patients of brain metastasis who presented to a tertiary care hospital in eastern India. 


\section{AIMS AND OBJECTIVE}

1. To study the various radiological presentations in cases of intracranial metastases.

2. To evaluate the various MRI presentations of metastasis among the patients.

\section{MATERIAL AND METHODS}

The present cross sectional study was carried out amongst 32 patients who had been visited tertiary care hospital in Kolkata with radiological features suggestive of metastatic lesion. Every patient had confirmation by biopsy of the lesion. The most common features that were compared are - age of patient, number of lesions, site of lesions, size of lesions, perilesional edema, margins, shape, T1, T2,FLAIR, GRE, DWI, MRS, skull lesions, scalp lesions,CT SCAN plain and contrast, histopathological findings.

The following inclusion criteria was used amongst the patients-

Patients with clinicoradiological diagnosis of metastatic brain tumors.

Patients with histopathological confirmation of diagnosis.

The following exclusion criteria was used among the patients-

Patients without histological confirmation of diagnosis.

Patient managed conservatively.

The study was approved from ethical committee of the institute and the study was carried out between August 2015 to July 2016. Based on these the cases were selected, observations were made and data was collected and tabulated.

\section{RESULTS}

In the current study there were 18 female cases and 14 male cases. The sex ratio,male: female ratio-7:9.
Number of lesion- Single: Multiple-6:3.

The mean age of diagnosis is 46.56years and range from 14 years to 65 years(Figure 1)

CT SCAN - 75\% patient had metastatic lesion appearing hyperdense on plain study and $25 \%$ had lesion hypodense on plain CT Scan (Figure 2a-b).

\section{MRI findings}

T1 wieghted images- In $75 \%$ of patient the metastatic lesions were hypointense to brain parenchyma on T1 weighted images and in $12.5 \%$ they were isointense and in $12.5 \%$ they were hyperintense (Figure 3a).

T2 weighted images- In $62.5 \%$ of patient the metastatic lesions were hyperintense to brain parenchyma on T2 weighted images and in $25 \%$ patients they were isointense and in $12.5 \%$ they were hypointense (Figure $3 \mathrm{~b}$ ).

Fluid Attenuated Inversion and Recovery (FLAIR)- In $87.5 \%$ of patients the T2 Flair hyper intensities were present which are suggestive of perilesional edema (Figure 3c).

Gradient Resonance Echo (GRE)- In 50\% of patient the metastatic lesions had shown blooming due intralesional calcification or haemorrhage (Figure 3d).

Diffusion Weighted Images (DWI)-In 75\% of patient the metastatic lesions had shown diffusion restriction (Figure 3e).

Contrast Images - On contrast images 37.5\% patients had homogenous contrast enhancement and 50\% had heterogeneous contrast enhancement and $12.5 \%$ had ring shaped enhancement (Figure 3f).

MRS- On MR spectroscopy in 62.5\% showed Choline peaks and and suppression of $\mathrm{N}$ - acetylaspartate (NAA) peaks in voxel based spectroscopy. There was reduction in NAA: Creatinine ration and rise in Choline: Creatinine and Choline: NAA ratio in the metatstatic lesions. Lactate peak was shown among $37.5 \%$ patients.(Figure $3 \mathrm{~g}$ ).

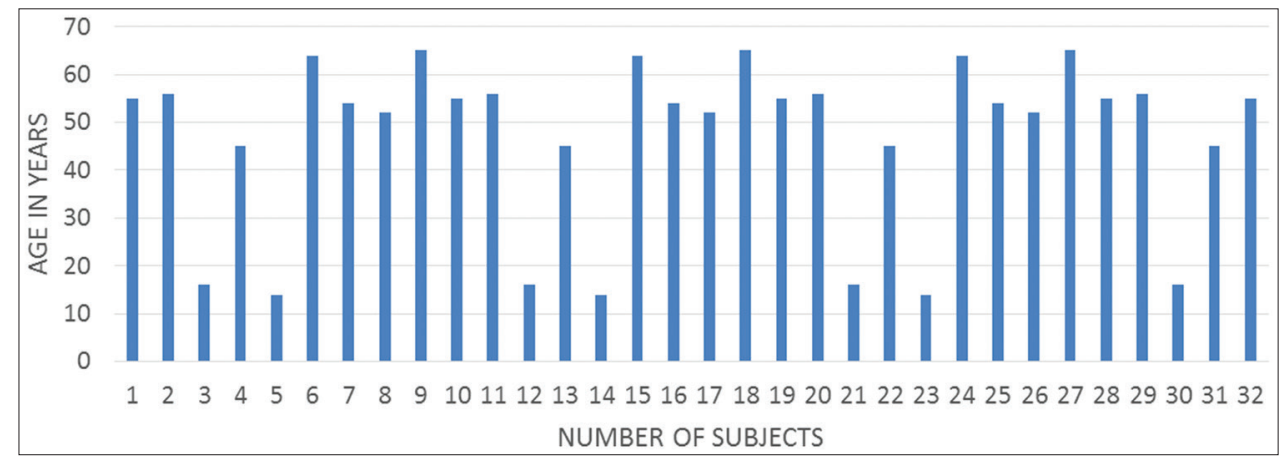

Figure 1: Showing age distribution among subjects 
Skull \& Scalp involvement - In 25\% patients had involment of skull bone and scalp skin involvement (Figure 4).

Biopsy - In 75\% the metastatic lesion turned out to be metastatic carcinoma with maximum cases of metastasis carcinoma lung followed by carcinoma breast and than by papillary thyroid carcinoma, adenocarcinoma from intestine, $12.5 \%$ turned out to be melanoma and $12.5 \%$ were metastatic deposit from leukemia in the orbit (Figure 5).

\section{DISCUSSION}

\section{Age incidence}

Metastatic disease in our series had age range from 14 years to 65 years. Mean age was 46.56 years. And maximum number of cases presented in $5^{\text {th }}$ and $6^{\text {th }}$ decades of life (Figure 1 ).

Stelzer KJ suggested an incidence rate of approximately 10 per 100,000 population and in the United States it was estimated that over 100,000 patients develop brain metastases each year. ${ }^{1}$

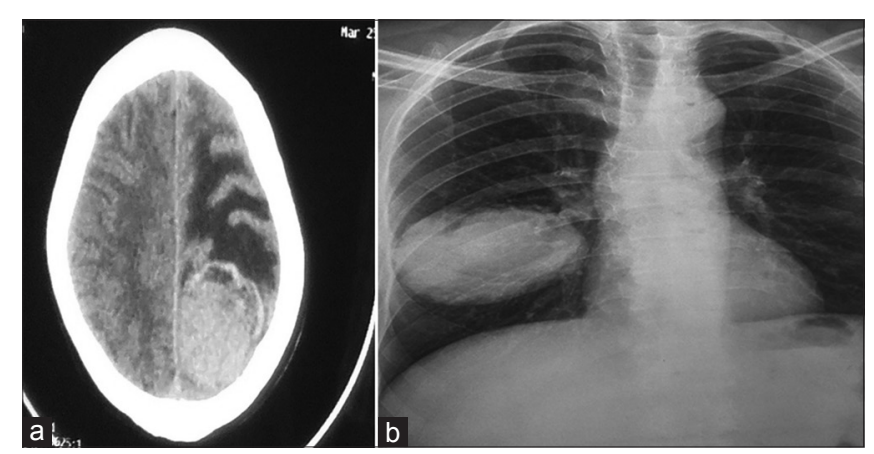

Figure 2: (a) Showing hyperdense mass on plain CT Scan in left occipital region most likely to be a metastatic lesion with suspected primary in lung. (b) showing mass in right lung lower lobe of same patient
Tabouret et al in there study found that the median age at diagnosis ranged from 57 to 63 years. Age at diagnosis was influenced by primary cancer. He observed that there was no correlation between the age of peak incidence of brain metastasis and the age of peak incidence of the corresponding primary, especially for breast cancer. Male patients contributed maximum number of cases in past years but recent trends show more female patients than male. ${ }^{2}$ According to studies by FJ Lagerwaad et al,Saha et al and Sarita Singh et al have also found similar age patterns. $3,5,6$

\section{Number of lesions}

F J Lagerwaad et al showed that number of lesions had prognostic significances. Different lesions at different site can have an overall impact over the clinical presentation, performance score, treatment and outcome. ${ }^{3}$

Lee in his study found that the distribution of metastases roughly follows the relative weight of and blood flow to each area of the brain. Thus, brain stem metastases (3-5\%), cerebellar metastases (10-15\%), and hemispheric metastases $(80-85 \%)$ arise approximately proportionately to the weight of these structures and blood circulation. In $50-85 \%$ of cases metastases are multiple, depending on the type of primary malignancy. Metastases from malignant melanoma, lung cancer, and breast cancer frequently lead multiple metastases. Renal cell carcinomaand colon carcinoma generally produce single metastases.

Fink KR and Fink JR showed that up to $80 \%$ of brain metastases occur in the cerebral hemispheres, $15 \%$ in the cerebellum, and $3 \%$ in the basal ganglia. Certain cancers may preferentially metastasize to the posterior fossa, including uterine, prostate, and gastrointestinal primary tumors. Occasionally, tumors may metastasize to the choroid plexus, ventricles, pituitary gland, or leptomeninges. Rarely, some

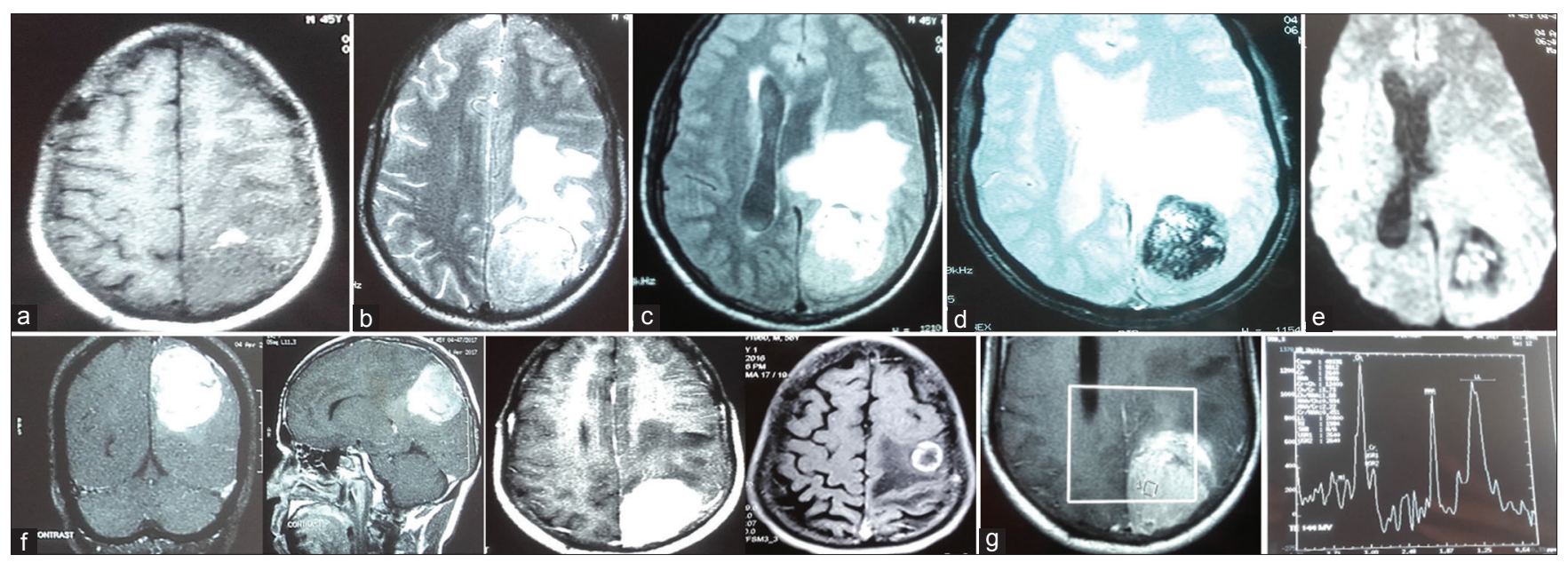

Figure 3: (a) Showing T1 weighted images with hypointense lesion. (b) showing T2 weighted hyperintense leison. (c) showing T2 Flair images with perilesional hyperintensities suggestive of edema. (d) showing GRE image with intralesion blooming. (e) showing diffusion restriction in centre of lesion. $(f)$ showing contrast enhancing lesion with dural enhancement and ring enhancement. (g) showing MRS images with Choline peak and lactate peak with diminished NAA peak and increased Choline to creatinine peak and Choline to NAA peak suggestive of malignancy 
malignancies including lymphoma may spread along or within the cerebral vessels. ${ }^{4}$

In the current study $62.5 \%$ metastatic lesions were in supratentoial region involving mainly Frontoparietal region. In $12.5 \%$ infratentorial and cerebellar region (Figure 6 ) and $12.5 \%$ involved the brainstem and $12.5 \%$ involved the orbit. The ratio of single to multiple (Figure $2 \& 7$ ) lesion in the current study was 6:3.

\section{Radiological imaging}

CT SCAN - 75\% patient had metastatic lesion appeared hyperdense (Figure2,4,6) on plain study and 25\% had lesion were hypodense on plain CT Scan.

Metastatic lesions may appear as a single or multiple lesions with or without surrounding edema. Based on its characteristics it can have a hypodense, isodense or hyperdense appearance on C'T Scan.

A hyperdense lesion on CT is suggestive of three differential diagnosis- Acute hemorrhagic metastasis, Melanin containing metastasis or calcification in metastatic

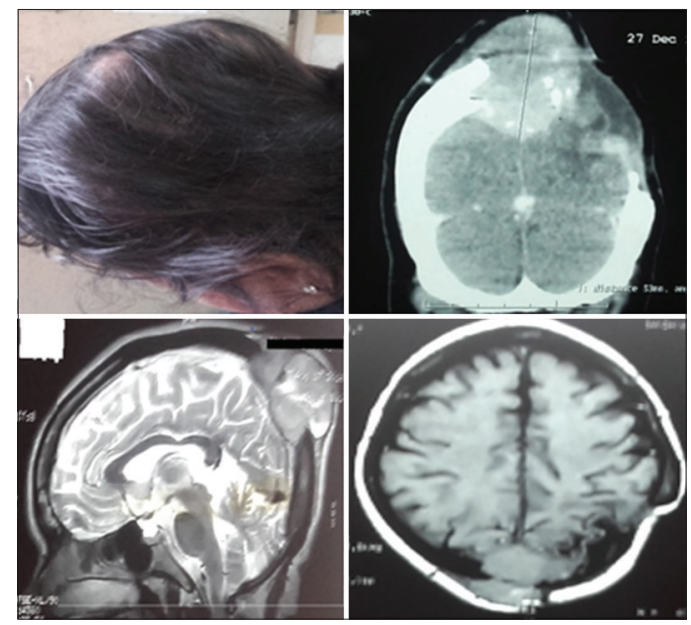

Figure 4: Showing a diagnosed case of papillary carcinoma thyroid with scalp mass and Ct Scan showing extensive skull bone destruction and MRI images suggestive of calvarial metastasis

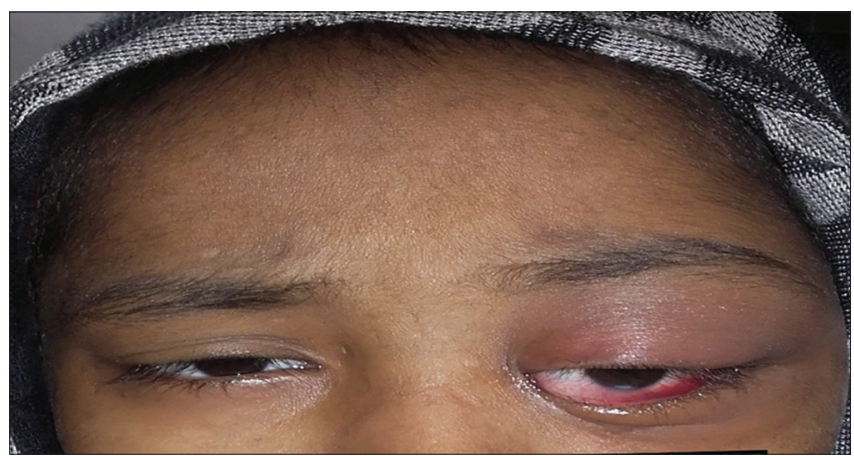

Figure 5: showing a diagnosed case of leukemia with orbital proptosis due to metastasis lesion. The third possibility is less likely than first two. ${ }^{4}$ On contrast administration,brain metastasis shows ring, nodular or solid enhancement. The advantage of CT Scan lies in fact that it is easily accessible, time saving and can also be used if MRI is not available or contraindicated. ${ }^{4,8}$

\section{Which modality is better CT or MRI}

MRI is having better sensitivity in screening brain metastasis. MRI is useful in cases when surgery or radiotherapy is planned and also CT is negative or inconclusive of diagnosis of ICSOL, whether lesion is single or multiple, neoplastic or nonneoplastic. The number of lesions can affect the treatment. ${ }^{1}$

Approximately $19 \%$ of patients with a solitary metastasis on contrast CT have multiple metastasis on Contrast Enhanced MRI.MRI is able to identify posterior fossa/ infratentorial lesions and also leptomeningeal metastatic diseases. Contrast images helps inidentification of lesions. The timing and dosage of imaging after gadolinium contrast is crucial in imaging. Delayed imaging (5 to 30 minutes) post contrast administration may help in detecting smaller lesions $(<5 \mathrm{~mm})$. Delineation of these lesions may be

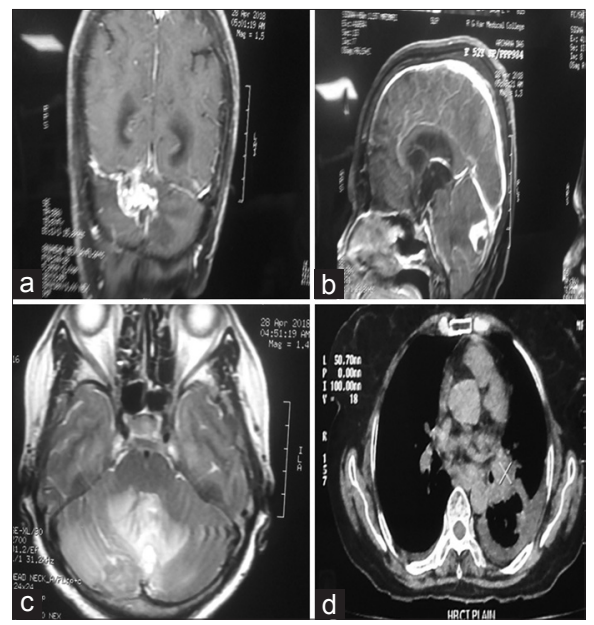

Figure 6: ( $a$ and b) Showing contrast enhancing infratentorial metastatic lesion lying in close vicinity to straight sinus and torcula, (c) showing axial T2 weighted image of same lesion, (d) showing HRCT of same patient showing lung mass in left basal region

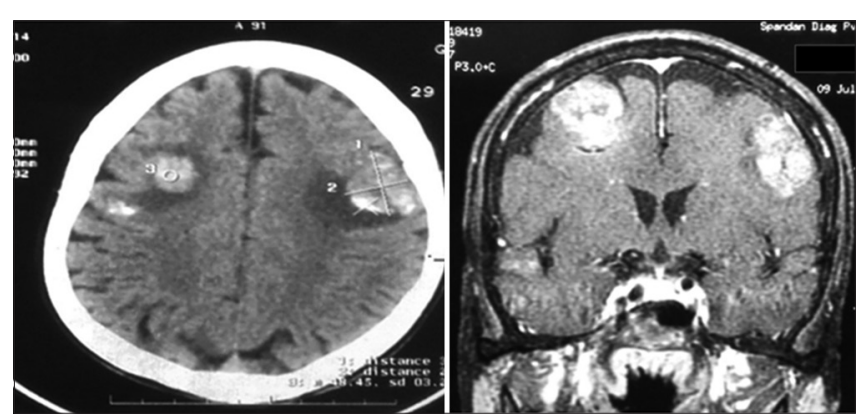

Figure 7: Showing plain CT scan and MRI T1 contrast coronal images with multiple metastatic lesion 
important in developing a therapeutic strategy for a patient and also number of lesions have prognostic significance. ${ }^{7,8}$

\section{MRI imaging overview}

Brain metastases are spherical, solid, or cystic, and well circumscribed lesions of various sizes at the corticomedullary junction, with varying amount of surrounding edema on MRI. The surrounding edema is usually vasogenic and disproportionately wide compared with the size of metastatic lesion. Metastases that are confined to the cerebral cortex, lesions measuring less than $5 \mathrm{~mm}$, may have little or no surrounding edema and can often be detected only on contrast enhanced images. Metastatic brain tumors rarely show prominent dural enhancement which is more likely due to reactive changes of the dura than tumoral invasion. ${ }^{7,8}$ Contrast administration is also important to distinguish nonneoplastic white matter disease (such as chronic microvascular ischemic disease, which does not enhance) from metastases. ${ }^{4}$

In current study, $75 \%$ of patient the metastatic lesions were hypointense to brain parenchyma on T1 weighted images (Figure 3a) and in 12.5\% they were isointense (Figure 4) and $12.5 \%$ they were hyperintense (Figure 7). In 62.5\% of patient the metastatic lesions were hyperintense to brain parenchyma on T2 weighted images (Figure $3 \mathrm{~b}$ ) and in $25 \%$ patients they were isointense and in $12.5 \%$ they were hypointense.On contrast images $37.5 \%$ of patients had homogenous contrast enhancement and $50 \%$ had heterogeneous contrast enhancement and $12.5 \%$ had ring shaped enhancement(Figure 3f).

\section{MRI features of metastasis}

On T1 weighted images, metastasis is usually iso or hypointense. On T2 weighted images are usually hyperintense and exhibit avid contrast enhancement. However, in case of melanoma (Figure 8c) hemorrhagic metastasis, can have different MRI presentation. On gadolinium contrast administration metastasis avidly enhance and can show homogenous enhancement or heterogenous enhancement like nodular or ring like pattern (Figure 3f), ${ }^{4,-10}$

In case of high protein secreting malignancies like Mucinsecreting tumors (gastrointestinal and genitourinary adenocarcinomas and at times lung) and hypercellular neoplasms, such as lymphoma (both primary and, metastatic), medulloblastoma/primitive neuroectodermal tumors, pineoblastoma, and neuroblastomas, the T2 weighted images can be hypointense due to high protein content within the lesion. Hemorrhagic metastasis constitutes $3-14 \%$ of intracranial metastasis and are common in melanoma, choriocarcinoma, renal, thyroid, lung, breast and germ cell tumors. It demonstrates hyper density on non-contrast CT and hyperintensity on T1 weighted images. T2 weighted gradient echo is the most sensitive technique for small hemorrhagic lesion detection. ${ }^{7,8}$

The malignant melanoma can be melanotic melanoma or amelanotic melanoma. The melanotic melanoma is hyperintense in relation to cortex on T1 weighted imagesdue to presence of paramagnetic effect of melanin and blood products and hypointense on T2 weighted images were as amelanotic melanoma is vice versa., ${ }^{710}$

\section{EDEIMA in metastatic tumors}

Vasogenic edema is always present surrounding the lesion, and is unrelated to lesion size. Massive edema surrounding contrast enhancing lesion size is more in highgrade primary brain tumors, but can also be a feature of

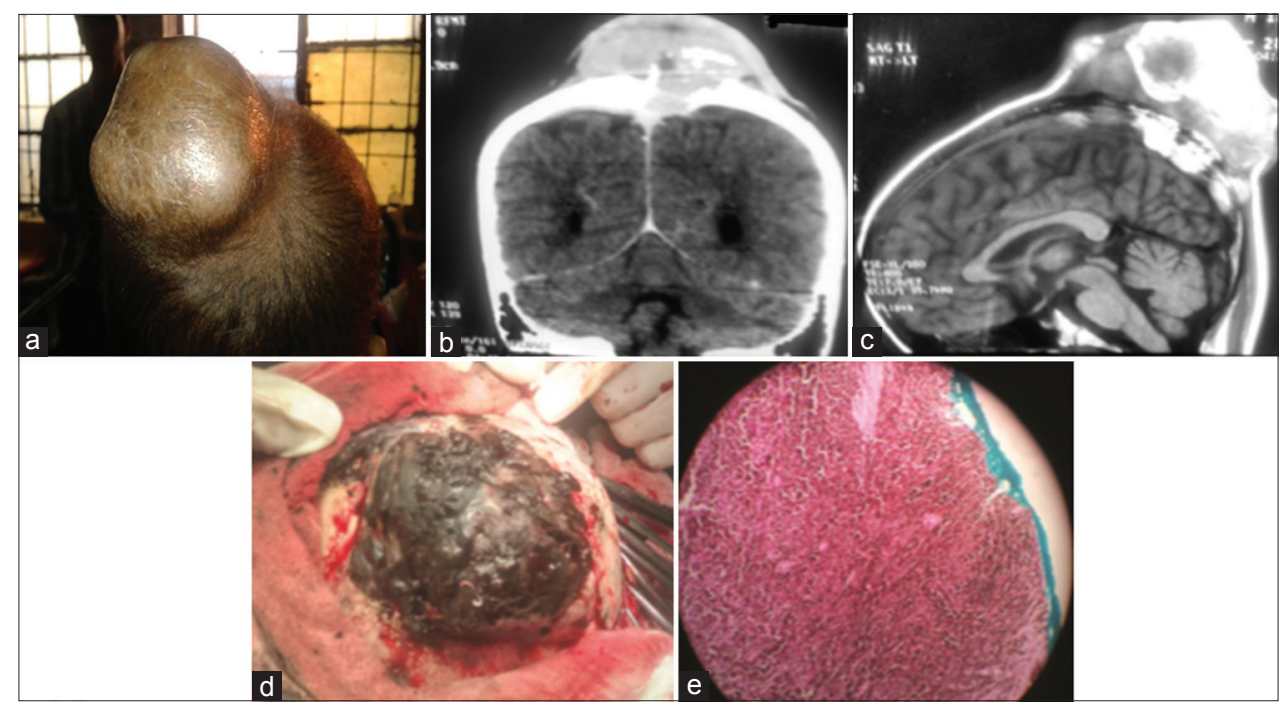

Figure 8: Showing a case of melanoma with intracranial metastasis. 8a- Scalp mass, 8b CT Scan showing Lytic skull lesion with mass, 8c Showing hyperintense T1 weighted MRI images, 8d showing peroperative photograph, 8e showing histopathological photograph. **b-e reprinted with permission from Dr Sandeep BV's article, Sandeep BV, S., Saha, S., Banga, M. and Ghosh, P. 2016. A rare case of intracranial malignant melanoma with an unusual presentation. Asian Journal of Medical Sciences. 7, 6 (Oct. 2016), 91-93. DOl:https://doi.org/10.3126/ajms.v7i6.15201 
metastatic lesion although metastases may also display little or no vasogenic edema. Small cortically based metastases may not demonstrate any visible edema. ${ }^{4}$

On Flair images (Figure 3c) 87.5\% patients the T2 hyper intensities were not suppressed on FLAIR sequences. High T2 signals is usually shown by metastasis, but also can indicate the cystic nature of lesion and also T2 hyperintensity not suppressed in FLAIR is suggestive of either perilesional edema, cystic necrosis in the lesion. ${ }^{7,8}$

GRE- In $50 \%$ of patient the metastatic lesions had shown blooming (Figure 3d). Gradient recalled echo (GRE) sequence can confirm both the presence of even small hemorrhage and prominent vessels. In combination of other sequences, it can provide some clue regarding the tissue of origin as hemorrhagic metastasis are common in melanoma, choriocarcinoma, renal, thyroid, lung, breast and germ cell tumors. ${ }^{7,810}$

\section{DWI}

On DWI images, In $75 \%$ of patient the metastatic lesions had shown diffusion restriction (Figure 3e).Diffusion Weighted Imaging(DWI) is based on Brownian movement of water in extracellular space and it is represented as increased signal intensity when there is obstruction in free water molecule movement and called as Diffusion restriction and degree of water movement is quantified in ADC mapping in which it is dark. Cause of diffusion restriction includes hypercellularity, cytotoxic edema, vascular insult like stroke. Metastasis show diffusion restriction due to surrounding perilesional edema which impairs intercellular free movement of water. ${ }^{7,8}$

MRS- On MR spectroscopy in 62.5\% showed Choline peaks and and suppression of $\mathrm{N}$ Acetyl Aspartate(NAA) peaks in voxel based spectroscopy. There was reduction in NAA: Creatinine ration and rise in Choline: Creatinine and Choline: NAA ratio in the metatstatic lesions. Lactate peak was shown among $37.5 \%$ patients (Figure $3 g$ ).

Choline is a measure of membrane turnover (resonates at $3.2 \mathrm{ppm}$ ) and increased with disorders that cause increase in cellular membrane turnover and in disease states that are associated with a hypercellularity. Creatine is a measure of cellular energyand relatively constant in the brain, so it is used as a reference (3.02 ppm). N-acetylaspartate (NAA) is a marker of neuronal viability (both concentration and integrity). NAA is found in mature neurons and neuronal processes (resonates at $2.02 \mathrm{ppm}$ ) and is reduced in disorders that destroy or replace neurons. Lactic acid (1.33 ppm) measures anaerobic metabolism. Lactic acid is elevated in acute stroke, recent seizure, and high-grade/necrotic neoplasms. Highly cellular lesions that outgrow their blood supply use up oxygen and rely on anaerobic glycolysis, with production of lactate. In addition, certain neoplasms have elevated glycolysis independent of oxidative metabolism. Myo-inositol is a cyclic sugar needed for cell growth and glucose storage. It has a prominent peak at $3.56 \mathrm{ppm}$. Located in glia (astrocytes), it indicates gliosis if elevated and is usually decreased in high-grade primary brain neoplasms. Lipid is seen in cellular breakdown ( 0.8 and $1.5 \mathrm{ppm})$ and is a marker of necrosis, as expected in high-grade malignancy both primary brain tumors and metastases. The general, nonspecific pattern for neoplasm is low NAA with high choline. In addition, lactate may be seen as neoplasms disrupt the normal glucose metabolism, with resulting hypoxia. Lipids may also be present in high grade neoplasms, secondary to necrosis. Common ratios evaluated in proton spectroscopy of the brain include the choline/creatine ratio and the choline/NAA ratio. Ratios may be calculated from either the maximum height of the peak, or from the area under the curve of the metabolite peak. ${ }^{7,8}$

Skull \& Scalp involvement - In 25\% patients had involvement of skull bone and scalp skin involvement. Skull involvement in metastatic lesion is more common in carcinoma lung, breast, thyroid kidney, and prostate, melanoma (Figure 4\&8). CT sacn may show focal skull bone distruction and rarely sclerotic as in cases of carcinoma prostate. Dural metastasis can either have thickened dura or enhancement which can be due to reactive involvement or neoplastic invasion from calvarial lesion (Figure 3f). Isolated dural involvemnts are rare. ${ }^{7,10}$

Biopsy - In $75 \%$ the metastatic lesion turned out to be metastatic carcinoma, $12.5 \%$ turned out to be melanoma and $12.5 \%$ were metastatic deposit from leukemia in the orbit.

Saha et al in their study observed carcinoma of the lung $(51.4 \%)$ followed by breast were the commonest primary that metastasizes to brain. ${ }^{5}$

FJ Lagerwaard et al and Tabouret et al,Sarita Singh et al, reported that the estimated global brain metastasis incidence was $9.6 \%$, in descending order arising from lung $(19.9 \%)$, melanoma $(6.9 \%)$, renal $(6.5 \%)$, breast $(5.1 \%)$ and colorectal cancer $(1.8 \%))^{2,3,8}$

EK Lee et al in his study reported that $50-85 \%$ of cases metastases are multiple, depending on the type of primary malignancy especially from malignant melanoma, lung cancer, and breast cancer. Renal cell carcinoma and colon carcinoma generally produce single metastases. ${ }^{7}$

\section{CONCLUSION}

A thorough and systematic approach to radiological investigation is crucial for proper identification of 
intracranial metastatic lesions. To sum up the clues of various radiological presentations can be a boon for proper diagnosis of lesion and also hint to tissue of origin. Moreover early identification of central nervous system metastasis can be very decisive in determining the therapeutic strategy and also prognosticating the patient outcome.

\section{ACKNOWLEDGEMENT}

None.

\section{REFERENCES}

1. Stelzer KJ. Epidemiology and prognosis of brain metastases. Surg Neurol Int 2013;4:S192-202.

2. Tabournet $E$, Chinot $O$, Metellus $P$, Tallet $A$, Viens $P$ and Goncalves A. Review: Recent Trends in Epidemiology of Brain Metastases: An Overview Anticancer Res November 201232 (11) 4655-4662

3. Lagerwaard FJ, Levendag PC, Nowak PJ, Eijkenboom WM, Hanssens PE and Schmitz PI. Identification of prognostic factors in patients with brain metastases: a review of 1292 patients. Int J Radiation Oncology Biol Phys 1999; 43(4):795-803.

4. Fink KR, Fink JR. Imaging of brain metastases. Surg Neurol Int 2013; 4:S209-S219.

5. Saha A, Ghosh SK, Roy C, Choudhury KB, Chakrabarty B and Sarkar R. Demographic and clinical profile of patients with brain metastases: A retrospective study. Asian J Neurosurg 2013; 8:157-161.

6. Singh S, Amirtham U, Premalata CS, Lakshmaiah KC, Viswanath L and Kumar RV. Spectrum of metastatic neoplasms of the brain: A clinicopathological study in a tertiary care cancer centre. Neurol India 2018;66:733-738.

7. Lee EK, Lee EJ, Kim MS, Park HJ, Park NH, Park SL, et al. Intracranial Metastases: Spectrum of Mr Imaging Findings. Acta Radiologica 2012; 53(10), 1173-1211.

8. Lignelli A and Khandji AG. Review of Imaging Techniques in the Diagnosis and Management of Brain Metastases. Neurosurg Clin N Am 2011; 22:15-25.

9. Zakaria R, Das $K$, Bhojak M, Radon M, Walker $C$ and Jenkinson MD. The role of magnetic resonance imaging in management of Brain metastases: Diagnosis to Prognosis, Cancer Imaging 2014; 14:8.

10. Sandeep BV, Saha S, Banga M and Ghosh P. A rare case of intracranial malignant melanoma with an unusual presentation. Asian Journal of Medical Sciences 2016; 7(6): 91-93.

\section{Authors' Contribution:}

SM- design of study, interpretation of images, darfting of manuscript; SD- design of study, collection of data, drafting of manuscript; SBV- Interpretation of images, proof reading; SKS-Concept and design of study, finalization of manuscript, proof reading; AN- collection of data, proof reading; DP- collection of data, proof reading.

Work attributed to:

Department of Neurosurgery, Nil Ratan Sircar Medical College Kolkata, West Bengal, India.

Orcid ID:

Dr. Shilpi Mucchoria- (1) https://orcid.org/0000-0001-5000-5772

Dr. Sourabh Dixit- (D) https://orcid.org/0000-0003-1085-7000

Dr. Sandeep B V- (10 https://orcid.org/0000-0003-4248-9062

Prof. Suniti Kumar Saha- (1) https://orcid.org/0000-0002-6217-9355

Dr. Abhishek Nadkarni- (1) https://orcid.org/0000-0003-2842-4756

Dr. Debjyoti Pathak- (1) https://orcid.org/0000-0002-8503-4618

Source of Support: Nil, Conflict of Interest: Nil. 